\title{
Handing Over the Keys: A Qualitative Study of the Experience of Automation in Driving
}

Conor Linehan ${ }^{1}$, Gillian Murphy ${ }^{1}$, Kieran Hicks ${ }^{2}$, Kathrin Gerling ${ }^{3}$ \& Kellie Morrissey ${ }^{4}$.

${ }^{1}$ School of Applied Psychology, University College Cork, Cork, Ireland

${ }^{2}$ School of Computer Science, University of Lincoln, United Kingdom

${ }^{3}$ Department of Computer Science, KU Leuven, Belgium

${ }^{4}$ Open Lab, Newcastle University, United Kingdom

Corresponding Author:

Conor Linehan, School of Applied Psychology, University College Cork, Cork, Ireland.

conor.linehan@ucc.ie

T: +353-21-490-4518 


\begin{abstract}
This paper presents a qualitative driving simulator study designed to understand the experience of giving up control to automated processes in semi-autonomous driving systems. The study employed an experience prototyping methodology, with 12 drivers (4 female) completing 2 sessions in a high fidelity driving simulator. Condition A simulated a normally functioning car, while Condition B simulated a semi-autonomous system that monitors driver behaviour and takes evasive action when danger is detected. The simulator experience was used to ground wider discussion of automation and the experience of driving, which was explored through a semi-structured interview. Results identify design challenges for autonomous driving systems; the loss of user agency and confidence, and handling the change between manual and automated control. Opportunities were identified; in augmenting rather than removing human abilities, and in providing new learning opportunities for drivers.
\end{abstract}

\title{
KEYWORDS
}

Driving, automation, experience prototyping, experience. 


\section{INTRODUCTION}

There is a much-discussed move towards automation in many areas of human activity (Hancock, 2014; Morozov, 2013). Motivated by an interest in safety, reliability and cost saving, human decisions, on every scale, are increasingly outsourced to algorithms trained on massive data sets. There have been many advances achieved through technical research in this field, such as efficiency gains in management of transport systems (Hoogendoorn, van Arem \& Hoogendoorn, 2014) and autonomous driving may have major positive impacts on policy and society, including reducing traffic, improving road capacity and reducing air pollution and accident risk (Milakis, van Arem \& van Wee, 2017). However, there is little qualitative research that concentrates on understanding the experience of receiving corrective advice from machines, or of being removed from the decisionmaking loop based on algorithmic judgement. Moreover, while the automation of vehicle control has drawn a great deal of media interest in recent years, little research has sought to understand how interacting with such systems, may impact upon the experience and practice of driving.

A great deal of research has been carried out on the design and evaluation of driving aids, such as automated cruise control (Vollrath, Schleicher \& Gelau, 2011), and automated steering and braking systems for collision avoidance (Young, Birrell \& Stanton, 2011). Given the great demands placed by driving on our limited-capacity cognitive abilities (Murphy \& Greene, 2016; 2017), there have been concerted efforts to explore the potential benefits and pitfalls of automated driving from a human factors perspective (Kyriakidis et al., 2017). Rarely does this research seek to understand the effect on the user experience of being monitored, advised, and/or overridden, by machines. This is surprising, since research in the field of Human-Computer Interaction (HCI) has long identified the importance of the sense of autonomy and agency to the experience of interacting with a system (Limerick, Coyle \& Moore, 2014). Moreover, the automobile itself has long represented a symbol of freedom and autonomy, thus attempts to limit or remove driver autonomy may have particularly negative consequences in this specific context (Steg, 2005).

This paper qualitatively examines participants' experience of having their driving monitored, evaluated, and having their control taken away. In the following sections, we will first discuss contemporary conceptions of the benefits of automation in driving. We present a brief review of previous studies identifying those relevant to user experience, where available. An experience prototyping study is presented where participants experience automated driving aids in the context 
of a driving simulator, before engaging in a semi structured interview designed to elicit experiences relevant to the simulated system, and to driving more generally. A set of considerations for the design of future systems is presented.

The novel contribution of this work lies in; 1) the in-depth, qualitative exploration of participant experiences and values related to interacting with an automated driving system, leading to 2) the publication of a set of experience-centered considerations that may help inform the design of semiautonomous driving systems. Through data gathered via a methodology that is novel in the context of studies of automated driving, we provide further support for design challenges already identified in the literature, such as the handover between machine and human control, and the loss of human agency. Moreover, we identify new opportunities and priorities for the design of autonomous driving systems, such as the importance of augmenting rather than bypassing human abilities, and of providing learning opportunities to drivers.

\section{LITERATURE REVIEW}

The goal of this paper is to understand how people experience the process of handing over control of driving to an automated system. This question can be answered in a number of different ways; for example, there is a cognitive component of that experience (do drivers understand when they are in control and when the system is in control, does automation cause distraction and error?), as well as a more values-related aspect (how does automation affect our experience of autonomy and agency? Do we actually want to buy cars that drive themselves?). While there is very little existing literature that addresses our exact research question, we can learn from related work on human factors in driving, and work examining the experience of interacting with autonomous systems more generally. First, we must define clearly what we are referring to as 'automated driving.'

\subsection{Envisioning the autonomous car}

In 2018, when this paper is being written, fully autonomous cars are being discussed heavily in the media and the research literature, but are not yet a reality on our roads. Thus, the work reported in this paper is somewhat speculative (Lawson, Kirman, Linehan, Feltwell and Hopkins, 2015) and oriented towards understanding potential implications of future technology. In this section, we will summarise the various visions of the future autonomous car presented by research and industry, while highlighting how existing semi-autonomous automotive systems fit within those visions. 
Contemporary arguments for the development of automated driving systems focus heavily on improvements in road safety, where future autonomous cars are predicted to significantly reduce or eliminate collisions (Gemtpton, Skalistis, Furness, Shaikh \& Petrovic, 2013; Jamson, Merat, Carsten \& Lai, 2013). They also address sustainability, where autonomous systems are envisioned to improve the flow of traffic, and the efficiency with which fuel is used by vehicles (Milakis, van Arem \& van Wee, 2017, Young, Birrell \& Stanton, 2011). They also include the wish to lower the cognitive load on drivers, freeing up time for them to engage in secondary tasks while driving (Brookhuis, van Driel, Hof, van Arem \& Hodemaeker, 2009). The level of automation envisioned in cars of the future varies. There are a number of competing visions, as outlined by Van der Laan (van der Laan, Heino \& de Waard, 1997). It is important to define clearly what we are referring to as 'automated driving,' as it is a term that can cover a range of different technologies.

Full autonomy describes a vision for cars where the driver has no input on the control of the vehicle, beyond selecting a destination (Thrun, 2010). The automated system takes care of navigation, steering, progress, braking and avoidance of obstructions. Often, this vision also involves some form of networked communication between vehicles to facilitate co-ordination. Surprisingly little of the published research specifically mentions full automation as a goal, possibly due to the complexity of technical challenges involved (Brookhuis et al., 2009). However, there has recently been a lot of publicity about organisations such as Google and Tesla working on fully automated cars. Unfortunately, there is little research published yet on the experience of using these vehicles.

The majority of the published literature investigates some form of semi-autonomous driving systems. These can be split into two categories; those that are activated by the driver, and those that are machine-activated. Since we are interested specifically in understanding the experience of interaction with these systems, it is necessary to define the different ways that we can already interact with these systems. Automated Cruise Control (ACC) has received a lot of attention from researchers (see Stanton \& Young, 2005 for a review). These systems are initiated by drivers, and are used to maintain progress on motorways, much like a standard cruise control. However, they also monitor the speed of vehicles ahead and speed up and slow down on behalf of the driver. Some examples can also steer cars to ensure they stay within their lanes. Other examples of drivercontrolled systems include traffic assistance (Young, Birrell \& Stanton, 2011) and parking 
assistance (Trösterer, Wurhofer, Rödel \& Tscheligi, 2014). These systems are already in use in some commercially available vehicles.

Research has been carried out on systems that monitor ongoing driver behavior and intervene on the driver's behalf. These systems are typically designed to brake on behalf of the driver when an obstacle is detected on the road ahead (i.e., Young, Birrell \& Stanton, 2011). Auto-braking systems are seen as safety features, thus are non-optional. These systems are also currently commercially available in some luxury cars. Similarly, there is little published research that seeks to understand the driver's experience of giving up control to these systems, with existing research suggesting poor understanding of these systems amongst the general public (Shimazaki, Ito, Fujii \& Ishida, 2017).

\subsection{Human Factors in Assisted Driving}

In this section, we will discuss research into human factors relevant for the design of automated driving systems. It is notable that the majority of this work has studied Assisted Cruise Control (ACC), as they are currently the most widely commercially available systems. Human factors research is typically founded on cognitive psychology principles and investigates questions surrounding the impact of automated systems on drivers' cognitive load (Brookhuis et al., 2009), attention (Young, Birrell \& Stanton, 2011), and safety (Gempton, Skalistis, Furness, Shaikh \& Petrovic, 2013), as well as the ability of humans to recognise when there are problems with the technology (Helldin, Falkman, Rivviero \& Davidsson, 2013) or the likelihood of the technology to interrupt driving (Stanton \& Young, 2005). Findings of this research suggest that ACC can lead to lower max speeds and less breaking of speed limits (Vollrath, Schleicher \& Gelau, 2011), less cognitive load on drivers (Stanton \& Young, 2005), less stress, and less fatigue (Neubauer, Matthews, Langheim, Saxby, 2012). Thus, this assistive technology may have positive impacts upon both road safety and wellbeing of drivers.

However, studies of automation in cars have also demonstrated undesirable findings. For example, increasing automation does not necessarily reduce human error (Gempton, Skalistis, Furness, Shaikh \& Petrovic, 2013). Automation has been found to undermine driver engagement in the driving task (Neubauer, Matthews, Langheim \& Saxby, 2012; Stanton \& Young, 2005; Jamson, Merat, Carsten \& Lai, 2013), to cause slower response times (Vollrath, Schleicher \& Gelau, 2011), to increase stress and to induce fatigue (Greenlee, DeLucia \& Newton, 2018; Neubauer, Matthews, Langheim \& Saxby, 2012). Studies also found confusion in drivers over whether the system was in 
operation, and how the handover between human and machine control was handled (Merat, Jamson, Lai, Daly \& Carsten, 2014). There is clearly much work to be done understanding how to best design these systems to ensure safety and comfort (Kyriakidis et al., 2017).

The studies mentioned above were generally interested in cognitive or behavioural effects of automation. Some research has also focused on understanding the subjective experience of automated cars. For example, Van der Laan et al., (1997) investigated user acceptance and found that systems that restrict drivers control are less likely to be accepted. Lai et al., (2010) found that intelligent speed adaptation faced similar issues of driver acceptance. Young et al, (2011) found that congestion assistants, which take control in traffic, were not acceptable to users. Molnar et al., (2018) found that trust in automated technology was significantly higher among those who reported being comfortable with other drivers behind the wheel. Although clearly interested in user experience, these studies use short questionnaires and a very narrow range of questions. They identify problems with the user experience, but offer little insight on how to address those problems.

Of course, not everything in the human experience can be captured by cognitive psychology concepts and human factors research. It is important to acknowledge also the subjective human experience of interacting with technology, which encompasses emotion, values and lived experience (see McCarthy and Wright, 2003, for an in-depth discussion of the concept of experience as it relates to technology design). In work closely related to the current paper, Ramm and colleagues, take an experiential, qualitative approach to understanding driver-car interactions (Ramm, Giacomin, Robertson \& Malizia, 2014). The intention was to understand the felt experience of driving in order to inform interface design. They suggest that "some drivers appear to desire simpler, more 'natural' car interfaces." However, they point out that little research exists on what characteristics or features make a car feel natural or unnatural. They set out to understand opinions regarding current and potential future car interfaces, with results suggesting that participants value full control, direct connection, skill, comfort, usability, car as a copilot, proactive assistance with environment, intelligent sensing, car as a single intelligent being, and voice control. Participants mentioned the feeling of being in control as particularly important: "Many drivers said they were generally much happier to drive than be driven, and that they would dislike being driven by an automated car as much as they would by any person." However, while Ramm et al. were interested in car interfaces, they did not 
specifically investigate automation in the driving experience. In contrast, Large et al. (2017) conducted a longitudinal qualitative study of drivers' behavior during simulated automated driving. Participants drove for 30 minutes per day across 5 days, mimicking their daily commute. Participants were encouraged to bring along objects and devices they would use if they were travelling in such a vehicle and were generally willing to allow the vehicle to take control and quickly became immersed in secondary tasks. Thus our paper, seeks to build upon the work of Ramm et al. (2014) and Large et al. (2017), looking specifically at participants' direct experience of automated systems in cars.

The current study was inspired by curiosity over the importance of autonomy and agency in people's experience of driving. Automated systems necessarily take control away from the user. However, research has consistently identified user agency (the subjective feeling of being in control) as key to the experience of using any technological system (Coyle, Moore, Kristensson, Fletcher \& Blackwell, 2012). For example, Schneidermans seventh golden rule of interface design (Schneiderman \& Plaisant, 2005) suggests that designers should strive to create computer interfaces that "support an internal locus of control" and that users "strongly desire the sense that they are in charge of the system and that the system responds to their actions" (see Limerick, Coyle \& Moore, 2014). It would seem that a loss of agency should be deleterious to the driving experience. Research has examined the effects of automation on driver's experience of agency. For example, Stanton and Young (2005) suggest that automation undermines people's perception of being in control of the vehicle. Perceived control helps support attention and mitigates the effects of fatigue. Moreover, Neubauer et al., (2012) suggest that, given the choice, drivers use automation sparingly. While these studies examine the effect of automation on the user's sense of agency, they do so primarily through focused quantitative instruments. The current study adds to this literature through in-depth qualitative study of the experience of automation.

\subsection{Interacting with Everyday Intelligent Systems}

While there is little existing research on the interaction of people with autonomous cars, there is some very relevant work examining the experience of interacting with other everyday autonomous systems. For example, Taylor (2009) discusses how the quality of peoples' interactions with artificial intelligences depends on what they understand intelligent behavior to be in that particular context. A series of case studies are presented, which demonstrate how expectations of 'intelligence' 
vary widely when interacting with, for example, safety-critical systems and virtual pets. One conclusion from this work is that in order to develop useful intelligent systems, research must first be carried out to understand peoples existing experiences and expectations of intelligent action in that context. This is one of the key goals of the current paper, since there is little work that already fulfills this role with regard to autonomous driving systems.

Interestingly, Taylor also discusses the move in AI research away from an, 'old,' paradigm focused on building independently intelligent things, and towards a 'new' paradigm based around supporting human intelligence in action. Indeed, this move reflects research (discussed above), which identifies the importance to people of feeling agency in the experience of using any interactive system. Interestingly, the intelligent cars currently under development, at least according to media reports, appear to follow the 'old' paradigm. Thus, the current paper, in exploring agency in autonomous driving systems, will address the appropriateness of adopting either the old or new paradigm with these systems.

\section{STUDY: UNDERSTANDING THE EXPERIENCE OF AUTOMATED DRIVING}

Semi-autonomous systems may be in all new cars in the very near future and very little is currently understood about how they impact upon the subjective experience of driving. The current study aims to explore and highlight user experiences, in order to understand better how to optimize the design of these systems. Unfortunately, it is simply impossible to study this experience in a naturalistic setting without causing huge risk to participants. The use of high fidelity prototypes (i.e., modified vehicles) on closed roads could still be open to questions of ecological validity. Thus, we adopt an 'experience prototyping' method, and study the phenomenon using a purpose-built high-fidelity driving simulator, followed by discussion with participants.

\subsection{Experience prototyping}

This study adopts an experience prototyping methodology (Buchenau \& Suri, 2000; Wright \& McCarthy, 2008), a research method that enables participants and researchers to gain first-hand appreciation of existing or future technology through active engagement with prototypes. The method is appropriate for use in situations where the technology does not yet exist, or in early studies of technology that is prohibitively expensive or dangerous. Typically with this method, there 
is some form of orchestration involved to give the appearance to users of a fully functioning system. Researchers observe behaviour and carry out interviews as appropriate to understand participants' subjective experience of using that technology.

While the use of a simulator in this study could be questioned in terms of ecological validity, we remind readers that there is a long and valuable tradition, especially in the field of Human-Computer

Interaction, of using prototypes and simulations (and role playing, design fictions, etc.) to study interactive experiences that are not accessible through other means. Our study falls within that tradition and suffers the same limitations. However, the alternative is essentially to not study this interesting and timely phenomenon.

Crucially, our driving task was designed to provide drivers with a sense of what it would feel like to use these systems during the complex process of driving on public roads. The new technology takes action in the context of everyday practices such as overtaking parked cars, driving past unpredictable pedestrians and approaching junctions. These incidents are used as prompts in the subsequent interviews. The data analysed in the study is purely based on the interviews, which also draw heavily upon participants' experience of driving more generally. In other words, engaging in the simulator experience is intended to ground the discussion and to ensure that all participants had a similar understanding of possible automation technology.

[insert figure 1 about here]

\section{Figure 1. Full size driving simulator used in the study}




\section{METHOD}

\subsection{Materials}

\section{Driving Simulator}

The study was carried out in the Driving Simulator Laboratory at University College Cork, using STISIM software (stisimdrive.com). The lab consists of a full-size 5-door manual drive Volkswagen Polo surrounded by 3 large screens. LCD screens in the wing mirror, a projection screen behind the car and speakers inside the vehicle create a fully immersive experience (see Figure 1). Drivers have full use of the gears, pedals, speedometer, tachometer and other vehicle controls. Steering feedback is appropriately weighted.

\section{Driving Tasks}

Drivers undertook two driving tasks; one control drive where the car behaved normally and the driver had full control of the vehicle and one augmented drive where the vehicle appeared to take control during hazardous situations. The control drive was necessary as driving in a simulator is already an unusual experience, thus we wanted to ensure participants' discussion during interview did not represent merely reflections on the novelty of simulated driving. Prior to the control drive, participants were instructed to drive as they normally would, obeying speed limits and other rules of the road. The instructions given prior to the augmented drive were; "'This device is designed to monitor and promote safe driving. You should drive as well and safely as you can, paying attention to hazards and speed limits, keeping the car in a straight line. The device uses complex algorithms to calculate when it needs to take over from you, for your own safety. It may not take over at all, depending on the quality of your driving.'

Each drive took place in a simulated suburban area with intermittent towns. The drives were undertaken in dry, bright conditions and the vehicle drove down a two-way road, with occasional oncoming traffic. Speed limit signs displayed the speed limit $(60 \mathrm{~km} / \mathrm{h})$ though participants could select their own speed. The drives contained a number of hazards such as a vehicle suddenly pulling into the lane in front, a pedestrian crossing the road, a stop sign at an intersection, and a vehicle 
reversing out of a driveway up ahead. The two drives were very similar and featured the same hazards, though they were encountered in a different order and featured different vehicle and pedestrian models to reduce practice effects. During the control drive, participants needed to avoid these hazards or the vehicle would crash, displaying a cracked screen and pausing for a moment before restarting the drive back on the road.

During the augmented drive, the vehicle took control as the hazard was encountered, the accident was avoided and speed was reduced. The augmented drive was designed to give the illusion of a system that monitored the environment for hazards and, upon detecting one, took control of the vehicle from the driver; applying brakes and steering to avoid collision. In other words, this was a machine-initiated semi-autonomous system (see background section). In practice, this 'taking over' of control occurred at pre-specified points where unavoidable hazards appeared on the road ahead. The vehicle took control 9 times, regardless of the drivers' behavior. When a hazard was encountered, a brief tone was played to indicate to the driver that they were no longer in control of the vehicle. At this point, none of the car input mechanisms (steering, accelerator, brakes, clutch) had any effect on the vehicle behavior. When the vehicle was in control, the accident was avoided and speed was reduced. The vehicle remained in control for a period from 5 to 30 seconds (depending on the specific hazard) until a short bell tone was played to indicate that the driver was once again in control.

We acknowledge that this simulation does not perfectly replicate normal road conditions; it exaggerated danger in order to create contexts where automation was appropriate. The inclusion of a high rate of incidents was ultimately a pragmatic decision. We wanted participants to experience the loss of control in a number of different scenarios, and in situations of varying danger, in order to understand the many potential implications of the technology on driving. Moreover, we remind readers that we were not interested specifically in driver behaviour during the simulation. The simulated drive functioned as part of an experience prototyping procedure, serving as a means to ground the subsequent interviews. 


\subsection{Participants}

12 participants ( 4 women) participated in this study. The mean age was 39.33 years ( $S D=9.93$ ). Participants all held driving licenses and had been driving for an average of 19.6 years (SD $=9.01$ ). Participants were recruited via a message placed on a university staff e-mail list, plus through personal connections. The study was advertised as examining "new, state-of-the-art monitoring technology designed to prevent road accidents" The only screening criterion employed was that participants held a full driving license. The relevant university research ethics board approved the study, and all standard procedures were followed surrounding informed consent, right to withdrawal, privacy and confidentiality.

\subsection{Procedure}

Participants initially read and signed informed consent forms, before filling out a questionnaire on demographics and driving experiences. They spent five minutes in a "practice" drive in order to help them become accustomed to driving in the simulator, and to ensure they were confident with the seat position and the working of the manual gear lever. One of two possible drives was then presented, representing either the control or augmented condition, the order of which was counterbalanced across participants. Both drives lasted approximately 12 minutes. A five-minute break was given, before participants engaged in the remaining drive.

After the driving phase, the semi-structured interview was carried out. Researchers followed a topic guide, enquiring into; a) initial reactions to the experience, b) the loss of control, c) their experience of technology in their own car, d) appropriate forms of automotive automation, and e) being technologically monitored. Crucially, participants were not simply asked to speculate on potential future technology, they were guided to reflect on both the simulated experience, plus actual past experiences, feelings and meanings around driving and car controls (Buchenau \& Suri, 2000 .

\subsection{Analysis}

Participant interviews were transcribed from audio files and the data were combined into a spreadsheet ready for analysis. Units were defined as speaking turns, thus there was significant variance in the length of those units. In total, there were 644 units included in the analysis. An inductive thematic analysis was then conducted on the data set following the method outlined by Braun and 
Clarke (2006). This method was deemed appropriate as the experience of automotive automation is an emerging and poorly understood topic, for which limited relevant theory already exists. One researcher read all units a number of times, and category codes were initially identified, together with a description and examples of each code. Units could be labelled with up to two codes each. A second researcher, who had done all the transcription work, then independently applied the coding scheme to the data and adjusted the scheme as they saw fit. An online meeting was held in which the codes were discussed and refined, until agreement was reached on a total of 19 codes. The coding categories were then examined and cross-referenced with the data and further analyzed for overarching themes (Braun \& Clarke, 2006), which were identified and reviewed by both researchers. 


\section{RESULTS}

Data from all twelve participants were combined into one document, giving a total of 644 relevant units, sorted into 19 codes. These codes were further combined to identify six core themes; Everyday Experience of Driving, Feeling of Control, Trust, Usability, Usefulness, and Monitoring. Figure 2 illustrates the coding scheme.

[insert figure 2 about here]

Figure 2. Illustration of themes that emerged from data analysis.

\subsection{Everyday Experience of Driving}

Participants discussed their own everyday experience of driving in great detail through all interviews. This topic was elicited specifically through the interview protocol, as we felt it important to understand how participants perceived and valued driving as an experience. It would be difficult to interpret peoples perceptions of automation in driving without understanding in detail how they experience driving as a day to day experience.

Participants expressed that they found driving to be a generally enjoyable experience, rather than a chore or a problem for which they are seeking a solution. Many participants discussed how they often go for a drive out of choice, rather than necessity, and how they, for example, take different routes to and from the same destination;

"I love driving. Even on holidays like I kind of look to go somewhere where there's good driving roads," (P9)

Some participants discussed the specific physical features of the driving process that they found satisfying or enjoyable

"I wouldn't go to an automatic gear stick because I enjoy the fact that you know just the gear changes everything in that like that I just like that part of driving" (P5)

However, some participants did mention that day-to-day driving for work, or city driving is a much less enjoyable experience. 
Participants often voluntarily discussed their perceptions of their own driving ability, when discussing issues such as road safety. It is important to understand peoples' perception of their own competence in order to understand their position on adopting safety technology. The majority of participants were very confident in their own ability to drive safely and effectively, and, interestingly, suggested that they were far better than other drivers,

"I still believe I am the safest driver there" (P2),

Indeed, some participants specifically commented on the fact that people seem to have an unrealistically positive view of their own ability;

"it's like they say right, it's not that I'm a bad driver it's that everybody else is" (P10), "they all think they are fantastic." (P8).

Participants' discussed their experience with existing driving technology, which provides useful context to their views on automation in driving. Participants in our study reported using a wide range of driving aids, such as standard cruise control, different versions of automated cruise control, parking sensors, dynamic suspension adjustment for different road conditions, dynamic steering adjustment for city versus motorway driving. Thus, the majority of participants were aware of, and had experience with, some driving aids. Interestingly, the majority specifically chose not to use automatic transmissions.

It is also useful to understand the types of dangers participants previously experienced on the roads. Many participants discussed the experience of personally being in a serious road accident, or of having close friends or family members killed or seriously injured in a road accident. They described hitting cyclists with their cars, being hit by cars when walking, and accidents with farm machinery on small countryside roads.

"I actually had an accident I don't know if I could have stopped..."It was a drunk coming the other way" (P1),

Thus, our participants have been exposed to the dangers of the roads, plus a range of modern driving technologies, they enjoy driving and have confidence in their own ability. 


\subsection{Feeling of Control}

One of the key phenomena that we sought to understand in this study was our participant's feeling of agency in driving a vehicle, and their experience of having that control taken away from them through automated intervention. Unexpectedly, our participants frequently mentioned that feeling fully in control of a vehicle was key to feeling safe and feeling confident in their driving ability. In reflecting on the handing over of control to an automated system, participants suggested that they would "hate" it and that it would be "scary."

"I would have difficulty delegating control like that" (P7),

This finding was consistent across the majority of participants, and should be expected, based on the long history of $\mathrm{HCl}$ literature that emphasises that the subjective feeling of control or agency over a system is key to the effectiveness in using that system. However, there were also contrary opinions. For example, P2 was very positive, suggesting that when the car took over he would "just remove everything" (P2) from the controls and wait. Indeed, most participants made some comment about how they didn't mind giving up control in situations where they had not reacted quickly enough;

"the only time I was really relieved during the simulation was yeah when someone had dashed in front of the car... they like obviously saved this person's life, and I was like oh thank God" (P11).

It seems that the experience of losing control can be very negative for drivers, but that it may be acceptable if activated only in critical incidents.

In exploring why the experience of giving up control was so negative, participants commonly mentioned the uncertainty caused by the system. Specifically, participants complained about feeling confusion over why the system activated;

"there was one time when it took over and I don't actually know why" (P4)

"If there was danger I didn't see it, you know, just wondering, what did I miss here. That sort of feeling."

Indeed, participants often insisted that the loss of control to the automated system was not necessary. They sought clarification over the level of danger that they had just avoided. There was confusion over whether the intervention of the system meant that there was otherwise definitely going to be an accident, which the system had to intervene in order to avoid, or whether there was 
only a small chance of accident. This provides a very clear example of how communication and education can play a key role in the acceptance of these technologies. It seems crucial, when implementing this type of system, to provide a mechanism through which drivers can understand why intervention was necessary, and what level of danger they had avoided.

Participants also discussed the experience of resuming manual control of the car. Previous research has identified that the process of handing control back to the driver is particularly problematic (Merat, Jamson, Lai, Daly \& Carsten, 2014) causing confusion and uncertainty for drivers. Many of the issues reported by our participants reflect that previous work. Our participants mentioned being "happy again when I was driving again" (P1), but the majority raised problems with the handover between machine control and manual control,

"you would be a bit shaky thinking 'Jesus is it having to give it back to me now and I was just nearly after crashing should I be driving?'." (P5)

\subsection{Trust}

Most participants talked about issues related to trust in automated driving systems. A minority of participants expressed some confidence in the potential of this technology to improve road safety. This seems to derive from a lack of trust of other drivers:

"I'd give it a shot....you're kind of putting your trust in somebody anyway I don't know what their level of driving skill is.." (P8),

However, the majority of participants expressed hesitancy in trusting automated driving systems. They explained, at length, the complex challenges faced by engineers in constructing systems that can react safely and effectively to every situation that may arise on every type of road, and imagined specific situations where they doubted the usefulness of an automated system;

"I think the real world is often more complicated than our algorithms will tell us" (P1)

Participants speculated over feasibility of the complex technical problems involved, such as sensing and perception of the environment, making effective decisions, dealing with unforeseen events, and 
navigating illogical road layouts in old cities. Participants often contrasted the complexity of those tasks, with their own everyday experience of technology as being "flaky", or unreliable;

"what if it gets a blue screen of death while it's driving down the road you know" (P8)

One fascinating topic that emerged across a number of participants, and one which is of direct concern to designers and manufacturers, is the question of how people could learn to trust this type of technology. Specifically, many participants suggested that they would need to experience the technology in action in their own car before they would trust it:

"The proof would be in using it in a way. I can't conceptually say I believe in it without seeing it in action and experience it in action" (P6)

However, it is difficult to imagine a safe way for people to learn to trust a safety critical system, without placing them in some level of unnecessary danger. This is an issue that must be addressed specifically by designers in order for this technology to be accepted;

"I've done the same with ABS brakes.... just sped up to about 30 miles per hour and just foot to the floor on the breaks to see what it did" (P6)

“How do you know you have a bulletproof jacket till somebody shoots you right?”(P10).

Thus, participants were sceptical over the ability of automated driving systems to act reliably, and suggested that only personal experience would undermine this scepticism.

\subsection{Usability}

Participants discussed the usability of the autonomous system in our study. Participants discussed their overall discomfort in using the system, with the vast majority expressing annoyance, frustration or uneasiness. The poor usability of the system appears to derive directly from the loss of agency, discussed at length earlier;

"I'm like f*ck you technology I can do this" (P4).

Participants repeatedly mentioned how they wished to turn the system off so that they could concentrate on the driving task. Most worryingly, participants suggested that the intervention of the system undermined their confidence in their own ability to drive safely;

"I was kind of getting annoyed at myself as well just for not having perceived hazards" (P9). 
Previous research on assistive driving has often questioned the impact of automation on drivers' attention (Stanton \& Young, 2005; Brookhuis, van Driel, Hof, van Arem \& Hodemaeker, 2009), with concerns that use of automated systems could lead to a drop in driver vigilance and over-reliance on the technology. Surprisingly, participants in our study instead generally reported being more attentive while driving in the augmented condition

"because I didn't want it to take over" (P4)

It must be noted, however, that this hyper-vigilant behaviour may have been driven by the novelty of the task, and may dissipate after a longer history of using the system.

Participants discussed the problem of learning to use the system. None of the participants had previously driven a car with an automatic braking system or automatic parking system, while only a minority had used automated cruise control. Most participants emphasised that their reactions may be different given longer to adapt to driving with the automated system turned on,

"I'd say it's something you get used to really after a short while..... could get used to it.... it was just too short a time" (P1),

"it's something of course that could be learned I'm sure. To learn like that the machine is going to do more then what you grew up doing" (P11).

\subsection{Usefulness}

Participants discussed whether they would find an automated driving system personally helpful for their own day-to-day driving. There were mixed reactions to this question, which was curious, given the generally negative feeling of participants towards the technology over the entire course

of discussion. Most surprisingly, some participants were very accepting of the idea of the technology being available in their own car;

"Would I be in favour of it? Oh yeah 100\%." (P9),

"If it'd improve safety yeah" (P1),

These comments are difficult to understand, given the earlier concerns over feasibility, usability and interruption of the enjoyment of driving. On further inspection, participants were responding to this question as if those previous concerns had been addressed. It seems that the takeaway message 
is that people have grave concerns over whether it is possible to build systems that are reliable and safe. However, if those systems are demonstrated as providing significant safety improvements, then they will generally be accepting of them.

It must be noted that a minority of participants deemed the technology unnecessary or unwanted, regardless of how well it worked;

"I can see the safety benefits and I think everyone should have it, just not me" (P9).

Participants discussed whether they felt the technology would be helpful for other drivers. This represented a questioning technique designed to help participants take other peoples perspective. The majority of participants were overwhelmingly positive, much more so than they were about the benefits to their own driving;

"I presume elderly people or people who are umm people who have any kind of disabilities or whatever that slows them down" (P1),

"I'd like if everybody else had it that kind of thing....But I didn't have to have it" (P9).

Participants were asked directly whether they feel that automated driving safety technology is necessary. Once again, responses were surprising and took some time to understand. Participants overwhelmingly suggested that the technology is not necessary;

"realistically there aren't any disadvantages but I just don't really like it" (P4).

In other words, despite suggesting that there are benefits of such technology for themselves and others, they seem to reject the idea that they are necessary. In other words, participants asserted that they could manage without the technology. Perhaps, given that the technology is currently unavailable in all but very expensive cars, this response reflects participants' resilience and need to feel competent in driving safely in the meantime.

In considering the usefulness of driving aids, participants also suggested that automation has the potential to undermine a core attraction of the driving experience;

“You're becoming a passenger then aren't you? Why would I buy a car to be a passenger?" (P5), "I don't know if I'd buy a self-driving car because then I might as well take a bus" (P8).

There was much discussion over specific design features that participants suggest would improve the usability and trustworthiness of the automated driving systems. Many participants discussed 
the importance of an automated driving system being seen as an aid, to not prevent the driver from undertaking their normal actions in response to an on-road event, but to augment those actions, "if there was a car that breaks hard in front of you or something pulls out in front of you, you're still going to do you own thing. Now what you hope is that the system might be slightly ahead of you so might get that extra half second or something." (P6),

"for it to take over control seems a bit extreme. You know maybe it could just tell me, okay slow down, but to take over in certain scenarios, it felt more danger, it...it did more danger then good" (P11).

Interestingly, this wish for systems that allow for an augmentation of human abilities, rather than removal of human entirely from the control loop, mirrors a recent shift in scholarship that seeks to best understand and design human interaction with machine intelligence and autonomous systems (Taylor, 2009).

\subsection{Monitoring}

Participants discussed the experience of having their driving behaviour monitored by an autonomous system. They suggested that the experience of being monitored is largely positive in the context of driving. For example, they suggested that such monitoring could help drivers with their awareness or perception of hazards on the road ahead. Moreover, many participants saw the benefits of having in-car sensors monitoring driver's physiological states. One participant (P3) suggested installing sensors on the steering wheel to monitor and ensure that the driver is actually engaged with the driving task, and to take control away from them if they are not paying attention. P5 suggested monitoring whether drivers were tired in order to help avoid accidents caused by people falling asleep at the wheel. P8 suggested using eye tracking to judge whether a person is paying attention to the road, and using this to help decide whether an automated system must take control. Finally, P11 suggested that alcohol monitors would be useful to help cut down on drunkdriving.

While participants saw many potential benefits of monitoring technology in the context of driving, there were also some concerns raised over the privacy and confidentiality of the data generated by such monitoring. For example, they raised concerns over the possibility of insurance companies gaining access to driving data and adjusting premiums without properly informing drivers of how the data informs their decisions; 
"I need to know, say with the insurance company, what exactly they're monitoring, what are they tracking? Is it every trip you take in the car? Is that being recorded somewhere?" (P6).

Some participants mentioned that the technological monitoring of their driving felt like being judged by the system, and experienced this as very negative.

"I feel like it's judging me.... I don't like being cr*p at things" (P4),

"Kind of disappointment in myself..... a bad reflection on my own driving" (P9),

It seems that taking control away from drivers, even if only very occasionally, has the potential to undermine drivers' confidence, which could have long term negative impacts upon their enjoyment of driving and self-efficacy. Two participants also discussed their experience with a car from the 1980s that had a voice interface;

"Somebody got one of those cars that spoke to you with some kind of voice system in it ...his buddy had learned to hate it with a passion ...they get out of the car and the car said 'Lights on! Lights on! Lights on!' and he just went 'it's your f**king battery!' and just walked away!" (P6),

This finding reflects wider HCI work on the experience of receiving corrective from mobile systems, which has found that interfaces considered condescending by users are generally disregarded (Cramer, Evers, van Slooten, Ghijsen \& Wielinga, 2010). Systems that engender in participants the feeling of being watched and judged will not be viewed favourably, especially if the perceived benefits are not entirely convincing. Indeed, some participants also discussed how they felt like they were being punished by the system when control was taken away from them. In order to avoid perceived punishment from a system, it is much easier to avoid using that system, than to change your behavior in a consistent manner (Kirman, Linehan, Lawson, Foster \& Doughty, 2010). 


\section{DISCUSSION}

In this paper, we explore the impact of vehicle automation on driving experience with the goal of better understanding the experience of being monitored and giving up control to automated processes. An experience prototyping study found that the experience of automation in simulated driving was often negative, with participants reporting that automation undermined a number of core attractions of the experience of driving, such as the feeling of control and trust. Based on our findings we discuss challenges and opportunities for the design of automated driving solutions, and we generalize our findings regarding automation and monitoring beyond driving, reflecting upon the implications that such technologies have for people's daily lives.

Previous research has identified many concerns about the effects of automation on the driving experience. Automation has been found to undermine driver engagement in the driving task (Neubauer, Matthews, Langheim \& Saxby, 2012; Stanton \& Young, 2005; Jamson, Merat, Carsten \& Lai, 2013), to increase stress and to induce fatigue (Neubauer, Matthews, Langheim \& Saxby, 2012). In addition many studies (van der Laan, Heino \& de Waard, 1997; Lai, Hjälmdahl, Chorlton \& Wiklund, 2010; Young, Birrell \& Stanton, 2011) found that systems that restrict drivers control are less likely to be accepted. Our study found largely negative participant experiences, similar to that previous work. This negativity seemed to be derived largely from the reluctance of participants to trust the system sufficiently to give up control, but also from their existing enjoyment of the experience of driving and confidence in their own abilities. Furthermore, participants noted that the intervention of the system felt like a judgment and criticism of their ability to drive safely, and affected their confidence, something that may create long-term negative effects.

Many commercial examples of in-vehicle monitoring and feedback technologies exist on the market today. Previous studies have evaluated these in terms of their potential to support, or distract from, the task of driving but have paid less attention to the subjective experience of being monitored while driving. Our study found that participants were surprisingly positive about the monitoring of their driving behavior, seeing advantages in terms of learning to drive safer or more fuel efficiently, or getting lower insurance premiums. Indeed, our participants suggested that more invasive monitoring than currently exists may be beneficial for road users, advocating for sleepiness monitors, alcohol monitors and monitors of whether the drivers hands are on the wheels. Thus, 
while participants saw the direct intervention of in-car systems as problematic, they were enthusiastic about monitoring technology.

\subsection{Considerations for the Design of Automated Driving}

\subsubsection{Challenges}

A number of design challenges were raised in the current study. These challenges largely reflect findings in related recent work on automated driving. Firstly, problems were identified in the process of handing control from the machine back to the driver. These results reflect the findings of Gempton et al. (2013) work with automated military logistic vehicles and also Helldin et al., (2013) on people's ability to recognize problems with automated systems.

Secondly, significant challenges were identified in how people can best learn to use (usability), and to trust, automated driving systems, given that they are only useful in safety critical situations. Our participants insisted that they would only trust the systems if they had first-hand experience of them working, and reported a similar approach to their acceptance of aids like cruise control and ABS brakes. There is a challenge for manufacturers in facilitating these types of experiences in a way that does not present significant danger for people and property.

Thirdly, the removal of agency (feeling of control) from drivers at safety critical moments has the potential to undermine driver confidence in their ability to drive safely. Not only does this present safety concerns, but a system that behaves in this punitive manner runs the danger of being outright rejected by users. The easiest way to escape punishment is avoidance.

It must be noted that these three challenges reflect previous findings on automated driving systems. Indeed, it would be very strange to find entirely novel results in such a well-studied topic. However, we adopted a different methodology than those previous studies, a methodology influenced by the recent shift in Human-Computer Interaction research away from focusing on cognitive workload and towards a more phenomenological and emotional account of experience. The fact that, through this new methodology, some of our findings were similar to those available in the literature, serves to demonstrate the validity of our methods, and also to lend weight to those previous findings through a process of triangulation of research methods. Our work adds depth, richness and colour to those primarily quantitative previous studies on UX in driving. 


\subsubsection{Opportunities}

We note three specific opportunities for improving the design of automated driving systems based on the findings of our study. These opportunities are novel in the context of the studying the experience of automation in driving. Firstly, the major concern of our participants was being outright removed from the control loop (feeling of control) and becoming a passenger in the system during a critical event. Participants suggested that systems that augment driver responses, perhaps through enhancing braking pressure or modifying steering, would be more acceptable than removing them completely from control. Participants also suggested the use of monitoring technology to 'check' whether the driver is attending to the road before taking control would be useful (usefulness). In this case, drivers would more easily recognise and understand the value of the system. The most negative comments from participants were regarding instances when the system took control when it was not perceived as necessary, or when the driver had the situation under control. Interestingly, this suggested focus on augmenting human abilities, rather than removal of human entirely from the control loop, reflects recent scholarship on designing useful interactions between humans and autonomous systems more generally (Taylor, 2009). It appears that fully autonomous systems are generally no longer desirable, and this appears to certainly be the case in the context of driving.

Secondly, designers should ensure that systems can communicate clearly to the driver afterwards why they were activated (usability-learning). In our study, people were confused and frustrated when they didn't understand the reason for intervention, but much more accepting when the reason for intervention was obvious and the outcome was positive. Indeed, these machine interventions may in future provide valuable learning experiences for drivers, diagnosing and explaining any hazards that arose, and any issues with driver responses to those hazards. Simply explaining why activation was required may go some way to mitigating the negative effects of losing agency. Indeed, more generally, machine intelligence will do more harm than good for users if it hides useful information from them.

\subsection{Deploying Machines in Our Daily Lives}

With autonomous systems and monitoring solutions becoming pervasive in our daily lives, understanding the implications of the changing relationship between humans and technology is 
essential to determine risks and challenges. In this context, our considerations need to extend beyond autonomous vehicles. With research exploring the deployment of autonomous and monitoring technologies in the lives of some of the most vulnerable members of society, for example, older adults (Pino, Boulay, Jouen \& Rigaud, 2015), and people with disabilities (Shiomi, Iio, Kamei, Sharma \& Hagita, 2014) we need to arrive at a better understanding of the effects that such technologies have on people. Our results suggest that benefits to the human experience can be gained from keeping users in the control loop (feeling of control) as much as possible. Rather than a

vision of automated systems undertaking tasks entirely outside of the everyday consciousness of users, systems may be accepted more readily if they involve and augment the existing abilities of users as much as possible. Moreover, many automated systems, including safety critical systems, may offer fascinating learning opportunities to humans; removing them entirely from the loop would remove that opportunity.

\subsection{Limitations and Future Work}

Our work needs to be considered in the context of the following limitations. Firstly, there were only 12 participants in the study. A larger or more diverse group of participants may have produced different reactions. Indeed, there may be significant cultural differences in attitudes towards automation technology. The study was carried out in Western Europe, where assistive driving technology has received relatively poor uptake in comparison with North America and East Asia. The same study carried out in those regions may report very different findings. Indeed, recent work has found cross-cultural differences in attitudes to automated driving, with drivers in more developed countries less comfortable with their vehicle transmitting data (Kyriakidis, Happee \& de Winter, 2015).

Secondly, it could be argued that the findings are relevant only to the specific system implemented in our simulator. However, we suggest that the value of this work is not in the specific participant responses to individual simulated stimuli, but rather as a provocation to participants to reflect on their experiences and values with respect to driving and the impact that automation may have upon those. Future work will involve exploring issues of experience, agency and automation in other contexts, such as assistive care for older adults, and automated wheelchairs. This will help build a 
deeper understanding of how to best deploy automation to support safety, agency and wellbeing (Hancock, 2014).

In conclusion, it is clear that technologies that have the capacity to monitor and automate our daily lives are becoming pervasive. While these systems hold enormous potential to support our future growth, environment, wellbeing and safety, we need to understand and carefully consider the many challenges surrounding the specifics of their deployment in order to ensure that the end result promotes wellbeing and minimizes harm. Our paper contributes to a better understanding of subjective responses to automation and monitoring in the context of driving.

\section{ACKNOWLEDGEMENTS}

This study was not funded by an external grant. No financial interest or benefit has arisen from direct applications of this research. We thank all participants for their time. 


\section{REFERENCES}

Babaee, S., Shen, Y., Hermans, E., Wets, G., \& Brijs, T. (2014). Investigating Individual Driver Performance: Applying DEA on Simulator Data. In Practical Applications of Intelligent Systems (pp. 623-635). Springer, Berlin, Heidelberg.

Bell, G., \& Dourish, P. (2007). Yesterday's tomorrows: notes on ubiquitous computing's dominant vision. Personal and ubiquitous computing, 11(2), 133-143.

Berberian, B., Sarrazin, J. C., Le Blaye, P., \& Haggard, P. (2012). Automation technology and sense of control: a window on human agency. PLoS One, 7(3), e34075.

Blythe, M. (2014). The hitchhiker's guide to ubicomp: using techniques from literary and critical theory to reframe scientific agendas. Personal and ubiquitous computing, 18(4), 795-808.

Braun, V., \& Clarke, V. (2006). Using thematic analysis in psychology. Qualitative research in psychology, 3(2), 77-101.

Brookhuis, K. A., van Driel, C. J., Hof, T., van Arem, B., \& Hoedemaeker, M. (2009). Driving with a congestion assistant; mental workload and acceptance. Applied ergonomics, 40(6), 1019-1025.

Buchenau, M., \& Suri, J. F. (2000, August). Experience prototyping. In Proceedings of the 3rd conference on Designing interactive systems: processes, practices, methods, and techniques (pp. 424-433). ACM.

Coyle, D., Moore, J., Kristensson, P. O., Fletcher, P., \& Blackwell, A. (2012, May). I did that! Measuring users' experience of agency in their own actions. In Proceedings of the SIGCHI Conference on Human Factors in Computing Systems (pp. 2025-2034).

Cramer, H., Evers, V., van Slooten, T., Ghijsen, M., \& Wielinga, B. (2010, April). Trying too hard: effects of mobile agents'(Inappropriate) social expressiveness on trust, affect and compliance. In Proceedings of the SIGCHI Conference on Human Factors in Computing Systems (pp. 1471-1474). ACM.

Fogg, B. J. (2002). Persuasive technology: using computers to change what we think and do. Ubiquity, 2002(December), 5.

Gempton, N., Skalistis, S., Furness, J., Shaikh, S., \& Petrovic, D. (2013, July). Autonomous control in military logistics vehicles: Trust and safety analysis. In International Conference on Engineering Psychology and Cognitive Ergonomics (pp. 253-262). Springer, Berlin, Heidelberg.

Greenlee, E. T., DeLucia, P. R., \& Newton, D. C. (2018). Driver vigilance in automated vehicles: hazard detection failures are a matter of time. Human factors, 60(4), 465-476.

Hancock, P. A. (2014). Automation: how much is too much?. Ergonomics, 57(3), 449-454.

Helldin, T., Falkman, G., Riveiro, M., \& Davidsson, S. (2013, October). Presenting system uncertainty in automotive Uls for supporting trust calibration in autonomous driving. In Proceedings of the 5th International Conference on Automotive User Interfaces and Interactive Vehicular Applications (pp. 210-217). ACM. 
Hoogendoorn, R., van Arem, B., \& Hoogendoorn, S. (2014). Automated Driving, Traffic Flow Efficiency, and Human Factors: Literature Review. Transportation Research Record: Journal of the Transportation Research Board, (2422), 113-120.

Jamson, A. H., Merat, N., Carsten, O. M., \& Lai, F. C. (2013). Behavioural changes in drivers experiencing highlyautomated vehicle control in varying traffic conditions. Transportation research part $C$ : emerging technologies, 30, 116-125.

Kirman, B., Linehan, C., Lawson, S., Foster, D., \& Doughty, M. (2010, April). There's a monster in my kitchen: using aversive feedback to motivate behaviour change. In CHI'10 Extended Abstracts on Human Factors in Computing Systems (pp. 2685-2694). ACM.

Kyriakidis, M., de Winter, J. C., Stanton, N., Bellet, T., van Arem, B., Brookhuis, K., ... \& Reed, N. (2017). A human factors perspective on automated driving. Theoretical Issues in Ergonomics Science, 1-27.

Kyriakidis, M., Happee, R., \& de Winter, J. C. (2015). Public opinion on automated driving: Results of an international questionnaire among 5000 respondents. Transportation research part F: traffic psychology and behaviour, 32, 127-140.

Lai, F., Hjälmdahl, M., Chorlton, K., \& Wiklund, M. (2010). The long-term effect of intelligent speed adaptation on driver behaviour. Applied ergonomics, 41(2), 179-186.

Large, D. R., Burnett, G., Morris, A., Muthumani, A., \& Matthias, R. (2017, July). A longitudinal simulator study to explore drivers' behaviour during highly-automated driving. In International Conference on Applied Human Factors and Ergonomics (pp. 583-594). Springer, Cham.

Lawson, S., Kirman, B., Linehan, C., Feltwell, T., \& Hopkins, L. (2015, April). Problematising upstream technology through speculative design: the case of quantified cats and dogs. In Proceedings of the 33rd Annual ACM Conference on Human Factors in Computing Systems (pp. 2663-2672). ACM.

Limerick, H., Coyle, D., \& Moore, J. W. (2014). The experience of agency in human-computer interactions: a review. Frontiers in human neuroscience, 8.

Merat, N., Jamson, A. H., Lai, F. C., Daly, M., \& Carsten, O. M. (2014). Transition to manual: Driver behaviour when resuming control from a highly automated vehicle. Transportation research part F: traffic psychology and behaviour, 27, 274-282.

Milakis, D., Van Arem, B., \& Van Wee, B. (2017). Policy and society related implications of automated driving: A review of literature and directions for future research. Journal of Intelligent Transportation Systems, 21(4), 324-348.

Molnar, L. J., Ryan, L. H., Pradhan, A. K., Eby, D. W., Louis, R. M. S., \& Zakrajsek, J. S. (2018). Understanding trust and acceptance of automated vehicles: An exploratory simulator study of transfer of control between automated and manual driving. Transportation Research Part F: Traffic Psychology and Behaviour, 58, 319328. 
Morozov, E. (2013). To save everything, click here: Technology, solutionism, and the urge to fix problems that don't exist. Penguin UK.

Murphy, G., \& Greene, C. M. (2016). Perceptual load induces inattentional blindness in drivers. Applied Cognitive Psychology, 30(3), 479-483.

Murphy, G., \& Greene, C. M. (2017). Load theory behind the wheel; perceptual and cognitive load effects. Canadian Journal of Experimental Psychology/Revue canadienne de psychologie expérimentale, 71(3), 191.

Neubauer, C., Matthews, G., Langheim, L., \& Saxby, D. (2012). Fatigue and voluntary utilization of automation in simulated driving. Human factors, 54(5), 734-746.

Pino, M., Boulay, M., Jouen, F., \& Rigaud, A. S. (2015). "Are we ready for robots that care for us?" Attitudes and opinions of older adults toward socially assistive robots. Frontiers in aging neuroscience, 7.

Ramm, S., Giacomin, J., Robertson, D., \& Malizia, A. (2014, September). A first approach to understanding and measuring naturalness in driver-car interaction. In Proceedings of the 6th International Conference on Automotive User Interfaces and Interactive Vehicular Applications (pp. 1-10). ACM.

Reeves, S. (2012, May). Envisioning ubiquitous computing. In Proceedings of the SIGCHI Conference on Human Factors in Computing Systems (pp. 1573-1582). ACM.

Saxby, D. J., Matthews, G., Hitchcock, E. M., Warm, J. S., Funke, G. J., \& Gantzer, T. (2008, September). Effect of active and passive fatigue on performance using a driving simulator. In Proceedings of the Human Factors and Ergonomics Society Annual Meeting (Vol. 52, No. 21, pp. 1751-1755). Sage CA: Los Angeles, CA: Sage Publications.

Shimazaki, K., Ito, T., Fujii, A., \& Ishida, T. (2017). The public's understanding of the functionality and limitations of automatic braking in Japan. IATSS Research.

Shiomi, M., lio, T., Kamei, K., Sharma, C., \& Hagita, N. (2014, October). User-friendly autonomous wheelchair for elderly care using ubiquitous network robot platform. In Proceedings of the second international conference on Human-agent interaction (pp. 17-22). ACM.

Shneiderman, B., Plaisant, C., Cohen, M. S., Jacobs, S., Elmqvist, N., \& Diakopoulos, N. (2016). Designing the user interface: strategies for effective human-computer interaction. Pearson.

Simons, D. J., \& Chabris, C. F. (1999). Gorillas in our midst: Sustained inattentional blindness for dynamic events. Perception, 28(9), 1059-1074.

Stanton, N. A., \& Young, M. S. (2005). Driver behaviour with adaptive cruise control. Ergonomics, 48(10), 12941313.

Steg, L. (2005). Car use: lust and must. Instrumental, symbolic and affective motives for car use. Transportation Research Part A: Policy and Practice, 39(2), 147-162.

Taylor, A. S. (2009, April). Machine intelligence. In Proceedings of the SIGCHI Conference on Human Factors in Computing Systems (pp. 2109-2118). ACM. 
Thrun, S. (2010). Toward robotic cars. Communications of the ACM, 53(4), 99-106.

Trösterer, S., Wurhofer, D., Rödel, C., \& Tscheligi, M. (2014, September). Using a parking assist system over time: Insights on acceptance and experiences. In Proceedings of the 6th International Conference on Automotive User Interfaces and Interactive Vehicular Applications (pp. 1-8). ACM.

Van Der Laan, J. D., Heino, A., \& De Waard, D. (1997). A simple procedure for the assessment of acceptance of advanced transport telematics. Transportation Research Part C: Emerging Technologies, 5(1), 1-10.

Schleicher, S., \& Gelau, C. (2011). The influence of Cruise Control and Adaptive Cruise Control on driving behaviour-A driving simulator study. Accident Analysis \& Prevention, 43(3), 1134-1139.

Wright, P., \& McCarthy, J. (2008, April). Empathy and experience in $\mathrm{HCl}$. In Proceedings of the SIGCHI Conference on Human Factors in Computing Systems (pp. 637-646). ACM.

Young, M. S., Birrell, S. A., \& Stanton, N. A. (2011). Safe driving in a green world: A review of driver performance benchmarks and technologies to support 'smart'driving. Applied ergonomics, 42(4), 533-539.

\section{Notes on contributors}

Conor Linehan is a Lecturer in Applied Psychology at University College Cork, Ireland, where he is a member of the People and Technology research group. His research explores processes for designing and evaluating technology for the purpose of health and education. Conor holds a PhD in Psychology from Maynooth University.

Gillian Murphy is a Lecturer at the School of Applied Psychology in University College Cork, Ireland, where she is a member of the People and Technology research group. Her research examines cognitive performance in applied settings, in particular attention and memory in everyday tasks such as driving.

Kieran Hicks is a PhD student in Computer Science at the University of Lincoln, UK. His research sits at the intersection of Human-Computer Interaction and game development, and explores the impact of visual embellishment on user experience and behaviour in interactive systems.

Kathrin Gerling is an Assistant Professor at KU Leuven, Belgium. Her work falls into HumanComputer Interaction, Accessibility, and Physical Computing, with a focus on how playful interactive technologies can support well-being. Kathrin received a PhD in Computer Science from the University of Saskatchewan, Canada.

Kellie Morrissey is a research fellow at Newcastle University. Her research focuses on the potential for digital design and technology to help people live meaningful and connected lives within their communities. She carried out her PhD in the School of Applied Psychology, University College Cork 